\title{
An LTR-observer-based dynamic sliding mode control for chattering reduction ${ }^{\text {ts }}$
}

\author{
Min-Shin Chen*, Chia-Hung Chen, Fu-Yun Yang \\ Department of Mechanical Engineering, National Taiwan University, Taipei, Taiwan, ROC
}

Received 8 September 2005; received in revised form 23 June 2006; accepted 7 December 2006

Available online 6 April 2007

\begin{abstract}
Two important approaches to alleviation of control chattering in sliding mode control are the boundary layer control (BLC) and the dynamic sliding mode control (DSMC). The DSMC is superior to the BLC since in DSMC chattering is alleviated without sacrificing the control accuracy. However, the design of DSMC is more challenging because its sliding variable contains an unknown system uncertainty. This paper proposes a robust two-dimensional LTR observer for estimation of the state-dependent uncertainty in the sliding variable. This paper also shows, via simulation examples, that the DSMC can better reduce chattering than the BLC especially in noisy environments.
\end{abstract}

(C) 2007 Elsevier Ltd. All rights reserved.

Keywords: Control chattering; Sliding mode control; Boundary layer control; LTR observer

\section{Introduction}

Sliding mode control is robust with respect to system uncertainties through the use of switching control or variable structure control (Hung, Gao, \& Hung, 1993). However, chattering of the control signal has become the major obstacle to its applications in the real world. In the literature, the first approach to chattering reduction is the boundary layer control BLC (Burton \& Zinober, 1986; Slotine \& Sastry, 1983). In the BLC design, the boundary layer width plays two contradicting roles: on one hand, it has to be large to reduce the control chattering; on the other hand, it has to be small to achieve good control accuracy. When the requirement on the control accuracy is high, the BLC becomes ineffective in reducing the control chattering. This is especially true when the state measurements are corrupted with measurement noises. When the measurement noise is of a level larger than the boundary layer width, the high-frequency oscillations in the noise will be reflected and amplified in the control signal (see simulation examples below).

\footnotetext{
is This paper was not presented at any IFAC meeting. This paper was recommended for publication in revised form by Associate Editor teD Iwasaki under the direction of Editor Roberto Tempo.

* Corresponding author.

E-mail address: mschen@ntu.edu.tw (M.-S. Chen).
}

The second approach to chattering reduction is the dynamic sliding mode control DSMC (Bartolini, 1989; Bartolini, Ferrara, \& Usai, 1998; Bartolini \& Pydynowski, 1996; Sira-Ramirez, 1993), where an integrator (or any other strictly proper low-pass filter) is placed in front of the system to be controlled, as shown in Fig. 1. The time derivative of control input, $w=\dot{u}$, is treated as the control variable for the augmented system (the system plus the integrator). A switching sliding mode control $w$ is then designed for the augmented system. Fortunately, the switching action of $w$ is contained in the controller, which is normally implemented within a computer, and hence will not do any damage to the real system. The control input to the real system $u=\int w \mathrm{~d} t$ becomes chattering free since the low-pass integrator in Fig. 1 filters out the high-frequency chattering in $w$. The advantage of DSMC design is that control chattering is reduced by low-pass filtering, not by sacrificing the control accuracy since no boundary layer is used in the design of $w$. Hence, the mechanism of chattering reduction and that of accuracy control are decoupled in the DSMC design. Another advantage of DSMC is that it is better immune to the measurement noise since the low-pass filter $(1 / s)$ in Fig. 1 can to some extent filter out the noise contained in the signal $w$.

Despite its superiority to the BLC, the design of DSMC is challenging for the following reason. In the DSMC design, 


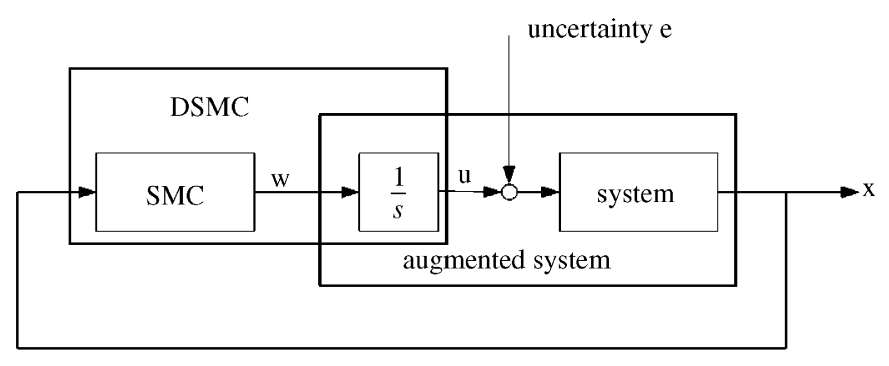

Fig. 1. Dynamic sliding mode control (DSMC).

the sliding variable is different from that in the BLC design since the augmented system in Fig. 1 is one dimension larger than the original system. As a result of this, the new sliding variable in DSMC contains an uncertainty term due to the external disturbance and/or parametric uncertainty. Evaluation of the new sliding variable in DSMC becomes difficult. This issue is not addressed in the early paper (Sira-Ramirez, 1993), but has been discussed by Bartolini in his series of works. In Bartolini (1989), a variable structure estimator is proposed to estimate the sliding variable in DSMC, but it must assume $a$ priori that the system state is uniformly bounded before proving the system stability. In Bartolini and Pydynowski (1996), a onedimensional observer is proposed to estimate the sliding variable, but stability is guaranteed only if a differential inequality with bounded coefficients is satisfied. The most recent approach (Bartolini et al., 1998) attempts to solve the problem based on a bang-bang optimal control without using any observer. But it is implementable only if one can detect the sign change of the derivative of an accessible signal. Finally, note that the sliding differentiator (Levant, 1998) approach for estimation of the sliding variable in DSMC also has to assume a priori a bounded trajectory.

To overcome the problem of sliding variable estimation in DSMC, this paper proposes using a two-dimensional LTR observer (Doyle \& Stein, 1979). The LTR observer, which was originally proposed for gain and phase margin recovery for observer-based LQ control, finds new applications in this paper for the estimation of state-dependent uncertainties in a dynamic system. The method proposed in this paper is better than existing methods since it does not need the bounded trajectory assumption (Bartolini, 1989), nor the differential inequality condition (Bartolini \& Pydynowski, 1996), nor the detection of sign change of the derivative of an accessible signal (Bartolini et al., 1998).

To simplify the writing of complex mathematical expressions, the following notations will be used in this paper. First, the big $O(\cdot)$ : one writes $s(t)=O(v(t))$ if after some finite time $|s(t)| \leqslant M|v(t)|$ for some finite constant $M>0$. Second, the small $o(\cdot)$ : one writes $s(t)=o(v(t))$ if after some finite time $|s(t)| \leqslant \varepsilon|v(t)|$ where $\varepsilon$ is a positive number that approaches zero as $\pi$ approaches infinity in the observer Riccati equation (8) in Section 2. Third, given a time signal $u(t),|u|_{t}=\sup _{\tau \leqslant t}|u(\tau)|$. These notations are standard in the study of adaptive system stability (Narendra \& Annaswamy, 1989).

\section{Sliding variable design in DSMC}

Consider the DSMC design for a linear system with uncertainty:

$\dot{x}=A x+B(u+e), \quad e=\Delta a x+d$,

$y=C x$,

where $x \in R^{n}$ is the accessible system state, $u \in R^{1}$ is the scalar control input, and $e=\Delta a x+d$ is system uncertainty, in which $d$ is an unknown disturbance with known upper bounds $|d| \leqslant \bar{d},|\dot{d}| \leqslant \bar{d}_{1}$, and $\Delta a \in R^{1 \times n}$ uncertain parameters with known upper bounds $\|\Delta a\| \leqslant \bar{a}$. The pair $(A, B)$ is controllable. The row vector $C \in R^{1 \times n}$ is a design parameter chosen such that $(A, C)$ is observable and zeros of the system $(A, B, C)$ are all in the open left-half plane.

The structure of DSMC is depicted in Fig. 1, where an integrator is placed in front of the system, and $w=\dot{u}$ is treated as the control variable to suppress the uncertainty $e$. This strategy is sometimes called the first-order dynamic extension of the control input $u$. A switching sliding mode control for $w$ is then designed to eliminate the uncertainty's effects. Even though $w$ is chattering, the control input $u$ to the system will be chattering free because the high-frequency chattering is filtered out by the integrator, which acts as a low-pass filter.

In the DSMC in Fig. 1, the augmented system is one dimension higher than the original system due to the inclusion of the integrator. As a result, the relative degree of the extended system becomes $r+1$, where $r$ is the relative degree of the original system $(A, B, C)$. Hence, a new sliding variable for the augmented system is chosen as

$s=y^{(r)}+\lambda_{r-1} y^{(r-1)}+\cdots+\lambda_{1} \dot{y}+\lambda_{0} y$,

where $\lambda_{i}$ 's are chosen such that $s=0$ defines a stable ODE of $y$. The task of the control variable $w=\dot{u}$ is to drive the new sliding variable $s$ to (almost) zero in the face of uncertainties. However, the new sliding variable $s$ is difficult to evaluate because its first term $y^{(r)}$ contains uncertainties $\Delta a x+d$ :

$y^{(r)}=C A^{r} x+C A^{r-1} B(u+\Delta a x+d)$.

The other terms $y^{(i)}=C A^{i} x, i=0, \ldots, r-1$, in (2) have no such problems because they are decoupled from the uncertainties.

Since $y^{(r)}$ cannot be evaluated correctly based on $x$ and $u$, a robust LTR observer (Doyle \& Stein, 1979) is suggested below to estimate $y^{(r)}$, and hence $s$. Define a two-dimensional state

$q=\left[\begin{array}{c}y^{(r-1)} \\ y^{(r)}\end{array}\right] \in R^{2}$,

where the first component $y^{(r-1)}=C A^{r-1} x$ is accessible for evaluation given $x$, but the second component $y^{(r)}$ is not accessible due to the uncertainties in (3). In the sequel, an LTR observer is employed to estimate $q$ and hence the inaccessible second component $y^{(r)}$. By taking the time derivative of (3), 
one can show that $q$ satisfies the dynamic model,

state: $\quad \dot{q}=A_{2} q+B_{2}\left(C A^{r+1} x+C A^{r} B u\right.$

$$
\left.+C A^{r-1} B w+\Delta p\right) \text {, }
$$

output: $\quad y^{(r-1)}\left(=C A^{r-1} x\right)=C_{2} q$,

where $\Delta p$ is an unknown uncertainty:

$$
\begin{aligned}
\Delta p= & C A^{r} B(\Delta a x+d)+C A^{r-1} B[\Delta a(A x \\
& \left.+B[\Delta a x+u+d])+d^{(1)}\right],
\end{aligned}
$$

and system matrices

$A_{2}=\left[\begin{array}{ll}0 & 1 \\ 0 & 0\end{array}\right], \quad B_{2}=\left[\begin{array}{l}0 \\ 1\end{array}\right], \quad C_{2}=\left[\begin{array}{ll}1 & 0\end{array}\right]$.

The following robust LTR observer is suggested to estimate the state vector $q$ in (5):

$$
\begin{aligned}
\dot{\hat{q}}= & A_{2} \hat{q}+B_{2}\left(C A^{r+1} x+C A^{r} B u+C A^{r-1} B w\right) \\
& +L_{2}\left(y^{(r-1)}-C_{2} \hat{q}\right),
\end{aligned}
$$

where $L_{2}=Q C_{2}^{\mathrm{T}} / \mu, \mu>0$, and

$\left(I+A_{2}\right) Q+Q\left(A_{2}+I\right)^{\mathrm{T}}-Q C_{2}^{\mathrm{T}} C_{2} Q / \mu+\pi B_{2} B_{2}^{\mathrm{T}}=0$

with $\pi>0$ sufficiently large.

Lemma 1 (Doyle \& Stein, 1979). Since $\left(A_{2}+I, B_{2}, C_{2}\right)$ is minimum-phase, the solution $Q$ of the observer Riccati equation (8) satisfies $\lim _{\pi \rightarrow \infty} Q / \pi=0$.

Based on Lemma 2, one can now show that the proposed LTR observer achieves small estimation errors of $q$ by using a sufficiently large design parameter $\pi$ in the observer Riccati equation (8).

Theorem 2. The LTR observer (7) and (8) achieves a small estimation error $\tilde{q}=q-\hat{q}$ in the sense that $\lim _{t \rightarrow \infty}\|\tilde{q}(t)\| \leqslant$ $\varepsilon_{1}\|x\|+\varepsilon_{2}|u|+\varepsilon_{3}$ where $\lim _{\pi \rightarrow \infty} \varepsilon_{i}=0$, for $i=1,2,3$.

Proof. Define the state estimation error $\tilde{q}=q-\hat{q}$; it satisfies, via (5) and (7),

$\dot{\tilde{q}}=\left(A_{2}-L_{2} C_{2}\right) \tilde{q}+B_{2} \Delta p$.

Set $V=\tilde{q}^{\mathrm{T}} Q^{-1} \tilde{q}$ for the error dynamics (9), and calculate its derivative

$\dot{V} \leqslant-2 V-\frac{1}{\mu}\left\|C_{2} \tilde{q}\right\|^{2}-\pi\left\|B_{2}^{\mathrm{T}} Q^{-1} \tilde{q}\right\|^{2}+2\|\Delta p\|\left\|B_{2}^{\mathrm{T}} Q^{-1} \tilde{q}\right\|$.

The maximum of the last two terms in the above equation occurs when $\left\|B_{2}^{\mathrm{T}} Q^{-1} \tilde{q}\right\|=\|\Delta p\| / \pi$, with the maximum value being $\|\Delta p\|^{2} / \pi$. Hence,

$\dot{V} \leqslant-2 V-\frac{1}{\mu}\left\|C_{2} \tilde{q}\right\|^{2}+\frac{\|\Delta p\|^{2}}{\pi} \leqslant-V-\left(V-\frac{\|\Delta p\|^{2}}{\pi}\right)$.
From the last equation, $\dot{V}<-V$ as long as $V>\|\Delta p\|^{2} / \pi$; therefore, eventually one has $\lim _{t \rightarrow \infty} V(t) \leqslant\|\Delta p\|^{2} / \pi$. Using $V(t) \geqslant \underline{\sigma}\left(Q^{-1}\right)\|\tilde{q}(t)\|^{2}=1 / \bar{\sigma}(Q)\|\tilde{q}(t)\|^{2}$, one derives

$\lim _{t \rightarrow \infty}\|\tilde{q}(t)\| \leqslant \sqrt{\frac{\bar{\sigma}(Q)}{\pi}}\|\Delta p\| \leqslant \varepsilon_{1}\|x\|+\varepsilon_{2}|u|+\varepsilon_{3}$,

where the last inequality is obtained by noting that $\Delta p$ defined in (6) is a linear combination of potentially unbounded $x, u$, and bounded $d$ and $\dot{d}$. Finally, by quoting Lemma 2, one concludes that $\lim _{\pi \rightarrow \infty} \varepsilon_{i}=0$ for all $i=1,2,3$.

Since $\left|\tilde{y}^{(r)}\right| \leqslant\|\tilde{q}\|$, it follows from Theorem 2 that the estimate $\hat{y}^{(r)}$ of $y^{(r)}$ obtained from the LTR observer (7) achieves a small estimation error, whose magnitude can be controlled by the design parameter $\pi$ in the observer Riccati equation (8). Following the notations introduced at the end of Section 1, one can say that

$\tilde{y}^{(r)}(t)=o(\|x\|)+o(|u|)+o(1)$.

The new sliding variable $s$ in (2) for the DSMC can now be approximately estimated with the help of the robust LTR observer (7). The estimate of $s$ is obtained as

$$
\begin{aligned}
\hat{s} & =\hat{y}^{(r)}+\lambda_{r-1} y^{(r-1)}+\cdots+\lambda_{1} \dot{y}+\lambda_{0} y, \\
& =\hat{y}^{(r)}+\lambda_{r-1} C A^{r-1} x+\cdots+\lambda_{1} C A x+\lambda_{0} C x
\end{aligned}
$$

with $\hat{y}^{(r)}$ the second element of $\hat{q}$ in observer (7).

\section{Control design in DSMC}

In the previous section, one has introduced the design of sliding variable $s$ in DSMC and presented a solution to its evaluation problem via the LTR observer. One can now present the control design for the control variable $w=\dot{u}$ in Fig. 1, which aims to drive $s$ to almost zero,

$u=\int w \mathrm{~d} t=\int[\phi(\hat{s})+v(\hat{s})] \mathrm{d} t$,

where $\phi(\hat{s})$ is a nominal control for the situation when there is no system uncertainty:

$$
\begin{aligned}
\phi(\hat{s})= & \frac{1}{C A^{r-1} B}\left\{-C A^{r+1} x-C A^{r} B u-\lambda_{r-1} \hat{y}^{(r)}\right. \\
& \left.-\lambda_{r-2} C A^{r-1} x-\cdots-\lambda_{0} C A x-\sigma \hat{s}\right\}
\end{aligned}
$$

and $v(\hat{s})$ a switching control to suppress the adverse effects of system uncertainty:

$v(\hat{s})=-\frac{\delta(x, u)}{C A^{r-1} B} \operatorname{sgn}(\hat{s}), \quad \delta(x, u)=\rho_{1}\|x\|+\rho_{2}|u|+\rho_{3}$,

in which $\rho_{i}$ 's $(>0)$ are chosen large enough so that $\delta(x, u)$ is an upper bound of $|\Delta p|$ in (6).

To facilitate the stability analysis, introduce a state transformation for system (1),

$x=T\left[\begin{array}{l}z \\ \eta\end{array}\right], \quad T \in R^{n \times n}$. 
The first group of new state $z \in R^{r}$ is the external state (Marquez, 2003) consisting of output derivatives,

$z=\left[\begin{array}{c}z_{1} \\ z_{2} \\ \vdots \\ z_{r}\end{array}\right]=\left[\begin{array}{c}y \\ \dot{y} \\ \vdots \\ y^{(r-1)}\end{array}\right]=\left[\begin{array}{c}C x \\ C A x \\ \vdots \\ C A^{r-1} x\end{array}\right] \in R^{r}$,

where $r$ is the relative degree of system (1). The second group of new state $\eta \in R^{n-r}$ is the internal state or zero dynamic state satisfying

$\dot{\eta}=Q \eta+P z$

for some matrices $Q$ and $P$, where $Q$ is a square matrix whose eigenvalues are open-loop zeros of the system $(A, B, C)$ (Marquez, 2003). Since by design, the system $(A, B, C)$ has only stable zeros, $Q$ is therefore stable. Note that the state transformation (16) is introduced here only for the purpose of stability analysis; it is not required in the computation of the proposed control law.

The stabilizing property of the proposed control (13) is proved by the theorem below.

Theorem 3. The proposed DSMC (13) practically stabilizes system (1) with bounded control $u$, in the sense that the system state is asymptotically driven into a residual set around the origin, with the size of residual set approaching zero as the design parameter $\pi$ in the observer Riccati equation (8) approaches infinity.

Proof. In this proof, one denotes $\tilde{s}=s-\hat{s}$, where $s$ and $\hat{s}$ are as given in (2) and (12). Choose $V_{2}=\frac{1}{2} s^{2}$ and check its derivative under the control $w$ in (13),

$$
\begin{aligned}
\dot{V}_{2} & =s\left[C A^{r-1} B v+\Delta p-\sigma \hat{s}+\lambda_{r-1} \tilde{y}^{(r)}\right] \\
& =-\sigma s^{2}+s\left(\sigma+\lambda_{r-1}\right) \tilde{y}^{(r)}+s[-\delta(x, u) \operatorname{sgn}(\hat{s})+\Delta p],
\end{aligned}
$$

where one has used $\hat{s}=s-\tilde{s}$ and $\tilde{s}=\tilde{y}^{(r)}$ to derive the second equality. There may be two possible cases for the square brackets in the above equation.

Case 1: $|s| \leqslant|\tilde{s}|$ : In this case, it follows from $\tilde{s}=\tilde{y}^{(r)}$ and (11) that

$|s| \leqslant \varepsilon_{1}\|x\|+\varepsilon_{2}|u|+\varepsilon_{3}$.

Case 2: $|s|>|\tilde{s}|$ : In this case, $\operatorname{sgn}(\hat{s})=\operatorname{sgn}(s-\tilde{s})=\operatorname{sgn}(s)$. Eq. (19) then becomes

$$
\begin{aligned}
\dot{V}_{2} & \leqslant-\sigma s^{2}+s\left(\sigma+\lambda_{r-1}\right) \tilde{y}^{(r)}-|s|(\delta(x, u)-|\Delta p|) \\
& \leqslant-\sigma s^{2}+s\left(\sigma+\lambda_{r-1}\right) \tilde{y}^{(r)} \\
& \leqslant-\sigma|s|\left[|s|-\left(1+\frac{\lambda_{r-1}}{\sigma}\right)\left(\varepsilon_{1}\|x\|+\varepsilon_{2}|u|+\varepsilon_{3}\right)\right]
\end{aligned}
$$

where the second inequality results from $\delta(x, u)>|\Delta p|$, and the last inequality results from (11). From the last inequality, one concludes that after some finite time

$|s| \leqslant\left(1+\frac{\lambda_{r-1}}{\sigma}\right)\left(\varepsilon_{1}\|x\|+\varepsilon_{2}|u|+\varepsilon_{3}\right)$.

Judging from conclusion (20) in Case 1 and conclusion (21) in Case 2, one concludes that

$s=o(\|x\|)+o(|u|)+o(1)$.

From (2), one can write $y=1 / D(s) s$, where $D(s)=s^{r}+$ $\lambda_{r-1} s^{r-1}+\cdots+\lambda_{0}$ is by design a stable polynomial. Following this, the external state $z$ in (17) is related to $s$ by $z^{\mathrm{T}}=$ $\left[1 / D(s), s / D(s), \ldots, s^{r-1} / D(s)\right] s$. Since all transfer functions in this equation are proper and stable, it follows from (22) and Lemma 2.6 in Narendra and Annaswamy (1989) that

$\|z\|_{t}=o\left(\|x\|_{t}\right)+o\left(|u|_{t}\right)+o(1)$.

Since $Q$ in (18) is a stable matrix, quoting (23) yields

$\|\eta\|_{t}=o\left(\|x\|_{t}\right)+o\left(|u|_{t}\right)+o(1)$.

Finally, it follows from (23), (24), and the state transformation (16) that the system state $\|x\|_{t}=o\left(\|x\|_{t}\right)+o\left(|u|_{t}\right)+o(1)$. This equation can be re-arranged as

$\|x\|_{t}=o\left(|u|_{t}\right)+o(1)$.

Since $y=1 / D(s) s$ as stated earlier, one has $y^{(r)}=s^{r} / D(s) s$, where the transfer function $s^{r} / D(s)$ is proper and stable. It then follows from (22) that $y^{(r)}=o\left(\|x\|_{t}\right)+o\left(|u|_{t}\right)+o(1)$. Substituting this result into (3) shows that $u(t)=\left(y^{(r)}-C A^{r} x-\right.$ $\left.C A^{r-1} B \Delta a x-C A^{r-1} B d\right) /\left(C A^{r-1} B\right)=O\left(\|x\|_{t}\right)+O(1)+$ $o\left(|u|_{t}\right)$. This leads to $|u|_{t}=O\left(\|x\|_{t}\right)+O(1)+o\left(|u|_{t}\right)$. Rearranging the equation gives

$|u|_{t}=O\left(\|x\|_{t}\right)+O(1)$.

Substituting (26) into (25) yields $\|x\|_{t}=o\left(\|x\|_{t}\right)+o(1)$, implying that

$\|x\|_{t}=o(1)$.

This suggests that after some finite time the proposed control achieves $|x(t)| \leqslant|x|_{t} \leqslant \varepsilon$ with $\lim _{\pi \rightarrow \infty} \varepsilon=0$. In other words, $x(t)$ will eventually be driven into a small residual set around the state space origin, with the size of residual set approaching zero as the design parameter $\pi$ in the observer Riccati equation (8) approaches infinity. Finally, substituting (27) into (26), one concludes that the control input $|u(t)| \leqslant|u|_{t}=O(1)$ is uniformly bounded. The boundedness of $w=\dot{u}$ follows from a careful examination of (13)-(15).

It is emphasized that according to the statement of Theorem 3 , the final control accuracy is controlled by the LTR-observer parameter $\pi$ in (8), while the chattering reduction of the control signal $u$ is achieved by low-pass filtering. Hence, the mechanism of chattering reduction and that of accuracy control are decoupled in the DSMC design.

Notice that the conventional BLC is susceptible to measurement noise when the noise level is larger than the boundary 

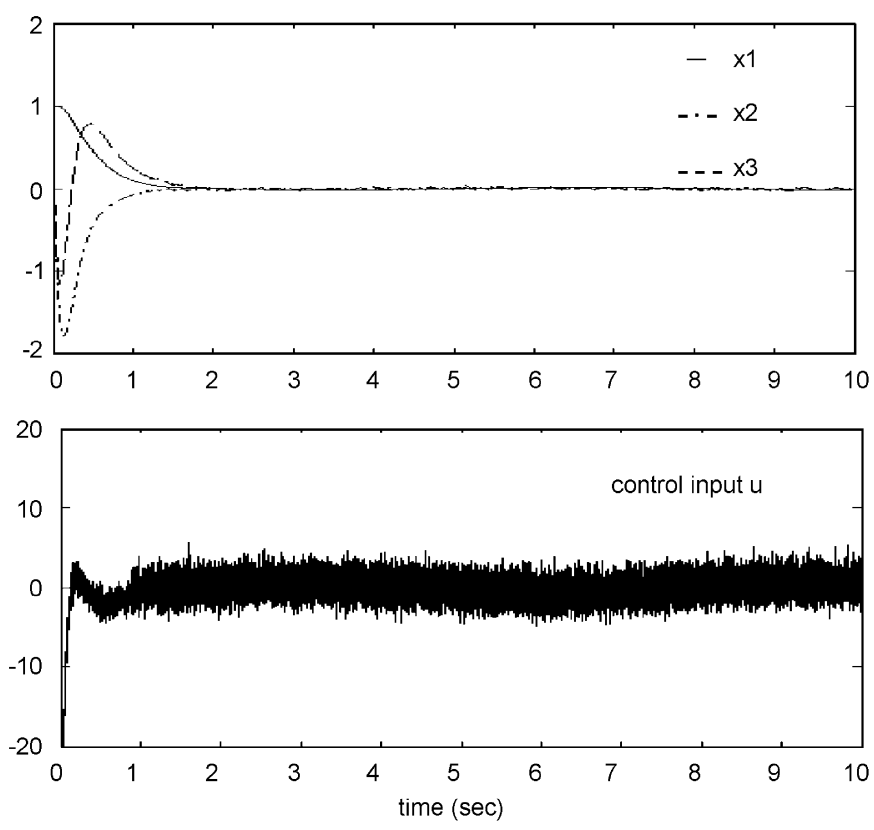

Fig. 2. Performance of boundary layer control.

layer width. When this happens, the high-frequency oscillatory noise will be reflected and amplified in the control signal. In contrast, in the DSMC, the low-pass filter $(1 / s)$ in Fig. 1 can to some extent filter the noise contained in the signal $w$, making the DSMC better immune to measurement noise. To compare how these two different controls perform in noisy environments, two simulation examples will be presented below.

Example $1(B L C)$. Consider a relative-degree-three system (1) with

$A=\left[\begin{array}{ccc}0 & 1 & -1 \\ -1 & -2 & 4 \\ 1 & -3 & -1\end{array}\right], \quad B=\left[\begin{array}{c}0 \\ 1.6 \\ 1.6\end{array}\right], \quad C^{\mathrm{T}}=\left[\begin{array}{l}1 \\ 0 \\ 0\end{array}\right]$,

$x^{\mathrm{T}}(0)=[1,1,1]$, and a disturbance uncertainty $e=\cos (t)$. One tests the conventional BLC with a small boundary layer width 0.05 . When the state measurement is noise free, the control signal will be smooth because of the use of a boundary layer. But when the state measurement is contaminated with an uniform noise with zero mean and standard deviation 0.001 , the results are quite different as shown in Fig. 2. The upper plot of Fig. 2 shows the system state (with noise removed), and the lower plot shows the control input. It is seen that although the system state is successfully regulated to almost zero in the face of unknown disturbance, the control signal has severe chattering due to the measurement noise.

Example $2(D S M C)$. The same system as in Example 1 is tested again for the proposed DSMC (13). The sliding variable in (2) is chosen with $\lambda_{2}=30, \lambda_{1}=300, \lambda_{0}=1000$. Other design parameters are $\delta(x, u)=35$ in (15), $\sigma=3$ in (14), $\mu=1$, $\pi=10^{8}$ in (8). The upper plot of Fig. 3 shows the time history of system state, which achieves almost the same performance
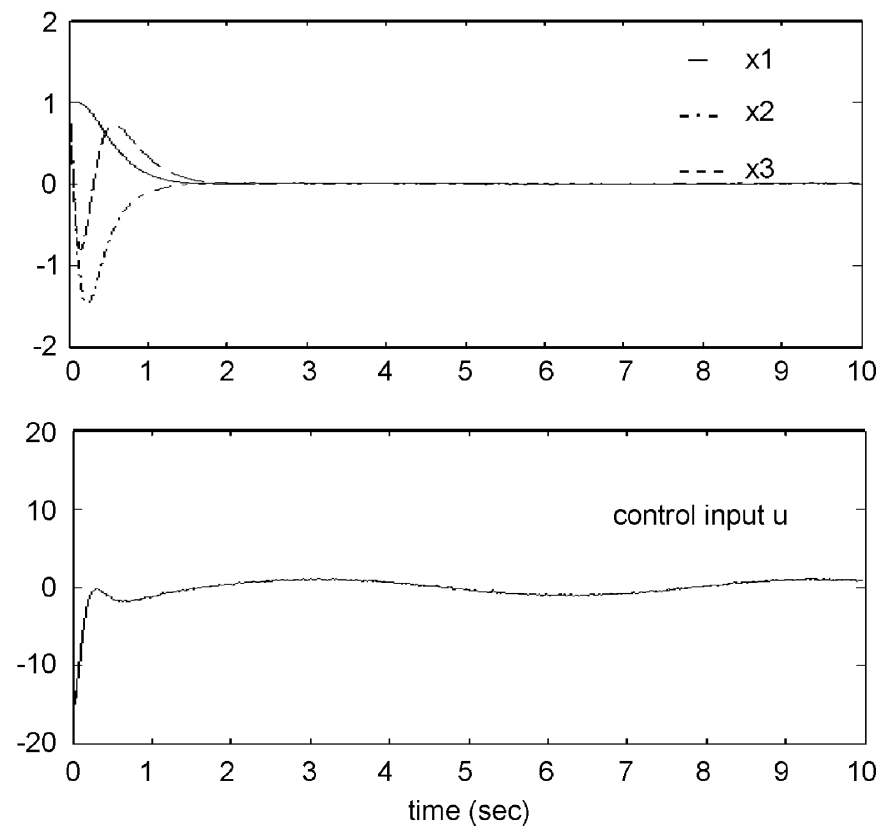

Fig. 3. Performance of dynamic sliding mode control.

as that with the BLC in Example 1. However, note from the lower plot of Fig. 3 that the dynamic sliding mode design has successfully removed the control chattering even in this noisy environment.

\section{Acknowledgement}

This work was supported by the National Science Council of the Republic of China under the Grant number NSC-94-2213E-002-080.

\section{References}

Bartolini, G. (1989). Chattering phenomena in discontinuous control systems. International Journal of Systems Science, 20, 2471-2481.

Bartolini, G., Ferrara, A., \& Usai, E. (1998). Chattering avoidance by secondorder sliding mode control. IEEE Transactions on Automatic Control, 43, 241-246.

Bartolini, G., \& Pydynowski, P. (1996). An improved, chattering free, V.S.C scheme for uncertain dynamical systems. IEEE Transactions on Automatic Control, 41, 1220-1226.

Burton, J. A., \& Zinober, A. S. I. (1986). Continuous approximation of variable structure control. International Journal of Systems Science, 17, 875-885.

Doyle, J. C., \& Stein, G. (1979). Robustness with observers. IEEE Transactions on Automatic Control, 24, 607-611.

Hung, J. Y., Gao, W. B., \& Hung, J. C. (1993). Variable structure control: a survey. IEEE Transactions on Industrial Electronics, 40, 2-22.

Levant, A. (1998). Robust exact differentiation via sliding mode technique. Automatica, 34, 379-384.

Marquez, H. J. (2003). Nonlinear control systems analysis and design. Hoboken, NJ: Wiley.

Narendra, K. S., \& Annaswamy, A. M. (1989). Stable adaptive systems. Englewood Cliffs, NJ: Prentice-Hall.

Sira-Ramirez, H. (1993). On the dynamical sliding mode control of nonlinear systems. International Journal of Control, 57, 1039-1061.

Slotine, J. J. E., \& Sastry, S. S. (1983). Tracking control of nonlinear systems using sliding surfaces with application to robot manipulator. International Journal of Control, 38, 931-938. 

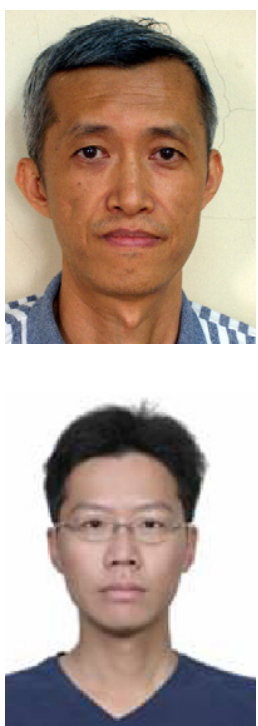

Min-Shin Chen received his B.S. degree in mechanical engineering from National Taiwan University, Taipei, Taiwan, in 1982, and both his Master and Ph.D. degrees in mechanical engineering from University of California, Berkeley, in 1986 and 1989, respectively. He is currently a professor with the Mechanical Engineering Department at National Taiwan University, Taiwan. His research interests include bilinear system control, sliding mode control, time-varying system identification, and robust observer design.

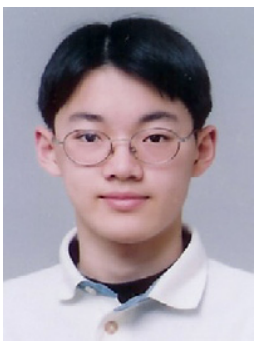

Fu-Yun Yang received his B.S. degree in mechanical engineering from National Cheng Kung University, Tainan, Taiwan, in 2003, and his Master degree in mechanical engineering from National Taiwan University, Taipei, Taiwan, in 2005. Presently, he is a Ph.D. candidate in Mechanical Engineering Department at National Cheng Kung University. His research interests include sliding mode control and sensorless motor control.

Chia-Hung Chen received his B.S. degree in power mechanical engineering from National Tsing Hua University, Hsinchu, Taiwan, in 2002, and his Master degree in mechanical engineering from National Taiwan University, Taipei, Taiwan, in 2004. His research interests include control applications, digital electronics, and DSP. $\mathrm{He}$ is presently working as a hardware research engineer in ASUSteK Computer Inc. 